\title{
A nuclear owner/operator perspective on ways and means for joint programming on predisposal activities
}

\author{
Michel Pieraccini* and Sylvain Granger \\ EDF Direction des Projets Déconstruction et Déchets (DP2D), 30 Avenue de Wagram, 75008 Paris, France
}

Received: 5 April 2019 / Accepted: 16 September 2019

\begin{abstract}
Nuclear decommissioning is a worldwide competitive market. It is also the main source of radioactive waste from the nuclear energy field. In order to reduce the waste volume it is necessary to sort the actual radioactive waste to be disposed of and to separate them from other materials that could be recycled. Since 2015, Electricité de France (EDF) has gathered the waste management and dismantling (WM\&D) projects, the related competences and human resources in the WM\&D field, in a dedicated directorate (DP2D) and a company group called Cyclife (including waste treatment facilities). Taking into account the experience gained by carrying out its own WM\&D projects as well as contributing to international cooperation, EDF considers that integrating collaborative research and development $(\mathrm{R} \& \mathrm{D})$ on pre-disposal and waste management could be carried out following four main objectives: (1) alignment of the application of regulatory frameworks through appropriate definition of criteria and rules for radioactive waste to enable sensible worldwide comparison of technics; (2) improvement of technical and organisational aspects of nuclear reactors decommissioning using a demonstrator facility to be in operation, at first for graphite reactors, by 2022; (3) development of new techniques to decontaminate/homogenize metallic materials through a dedicated recycling route. These technics will be implemented in a new treatment facility foreseen to be available by 2030; and (4) increased training of decommissioning operators with the help of new technologies. All these improvements are aiming, beyond technical and experimental aspects, at reducing environmental impacts of nuclear activities as well as preserving the radioactive disposal volumes, as they are considered by EDF as rare resources.
\end{abstract}

\section{Introduction}

As owner/operator of a nuclear fleet with the responsibility of the dismantling of already shutdown NPPs, Electricité de France (EDF) has acquired a sound and robust experience in waste management and dismantling (WM\&D). Since 2015, EDF has created in the WM\&D field a dedicated directorate (DP2D) and its holding called Cyclife. These new entities gather all corresponding projects, all necessary human skills and resources as well as technical means. Such new organisation enables DP2D to define and make applied the WM\&D strategy for the entire EDF Group and its partners.

The main objective of this new organization is to reinforce EDF's capacity and leadership in this field. It will allow the integration of R\&D aspects such as decommissioning operators' training, testing of innovative technologies (virtual reality, 3D simulation, remote operations) and development of technical demonstrators. Therefore, EDF intends to develop international collaboration directly through industrial cooperation and through

\footnotetext{
* e-mail: michel.pieraccini@edf.fr
}

participation in collaborative R\&D projects. These projects can be carried out under the umbrella of international organisations such as the European Commission, OECD/NEA or IAEA, in which EDF already contributes actively.

To define $\mathrm{R} \& \mathrm{D}$ programmes for improving radioactive waste management during pre-disposal periods, it is necessary to agree on the definition of "radioactive waste" and the various current or potential sources. There is now a global international agreement within the nuclear industry to call "radioactive waste" the ultimate status of a considered material that will require disposal. Thus, to assess the status of waste, all materials to be generated by nuclear activities should be precisely characterized and sorted out in order to reduce significantly the amount of actual radioactive waste to be disposed of.

The importance of reducing the radioactive waste volume has increased over the last decade since repositories are considered as rare resources to be preserved in order to limit environmental impacts as much as possible. This care about reducing waste volume is taken during the four steps of the lifetime of a nuclear facility: design, construction, operation and decommissioning. Then, as users of radioactive disposals, nuclear operators take on their 
responsibility, by enhancing their expertise as well as increasing their credibility by mastering techniques and costs. In addition, they contribute to avoid spoiling resources by disposing materials that can be reused or recycled.

Defining relevant $R \& D$ programs in WM\&D require that all aspects of predisposal activities are properly evaluated, considering the great diversity of type, nature and activity of waste generated by decommissioning as well as the involved quantities. Sufficient operational experience and feedback from existing practices help to identify remaining needs, commonly shared at the European or the international level.

\section{Strategy on waste management and decommissioning}

\subsection{Experience gained in performing decommissioning on an industrial scale}

In the last 10 years EDF has acquired experience in the decommissioning of four different types of nuclear reactors. This fruitful learning stage makes it possible to beneffrom some lessons learned. They can be summarized as follow: - For Pressurized Water Reactors (PWRs), which compose the current French operating fleet, the feasibility of the decommissioning process has already been demonstrated. The focus is now on optimizing the sequence of future operations and scenarios.

- For graphite reactors, the remote handling systems to dismantle these huge reactors are still under development. In parallel, the graphite waste management remains a major issue. Considering the size and complexity of this kind of reactors, dismantling design and operation will lead to long timeframes.

- A sound experience in waste management driven decommissioning is the cornerstone to successfully perform the dismantling of a nuclear reactor.

France can rely on a complete and efficient radioactive waste management system for nuclear waste produced during decommissioning. However, this system could be improved by addressing the issue of clearance levels to allow the recycling of ingots produced after the melting of very low-level radioactive metallic waste.

Specific care should be given to the use of terms for decommissioning For instance, the term "immediate dismantling" recommended by the IAEA, should not mean that dismantling should be hurried, irrespective of associated risks and costs, but that the decommissioning of the nuclear plant should start soon after its shutdown. This kind of misinterpretation could lead to counterproductive attitudes of counterparts including local stakeholders.

\subsection{Organisational issues}

Taking into account the experience gained, EDF, in 2015, decided to create and structure an entity devoted to decommissioning and waste management with three main objectives:
- support ongoing EDF dismantling worksites;

- prepare the decommissioning of the nuclear fleet in operation;

- become a major actor in the WM\&D market, which is emerging but already highly competitive.

This restructuring has been progressively conducted in three parallel phases:

- the creation of a dedicated directorate called DP2D gathering all corresponding projects, resources and skills. It reports directly to EDF Group Board;

- the creation of specialized subsidiaries providing waste treatment services in order to optimise the sorting and the volume of waste to be disposed of. In addition, it has aimed at increasing EDF's technical and financial mastery for its own needs (as a responsible owner/ operator of a world nuclear fleet) or those of its partners/ customers (who trusts and relies on EDF capacities and skills worldwide to preserve and develop their own competitiveness);

- the establishment of industrial partnerships regarding innovative and key technologies or targeted geographic areas.

In order to take advantage of the ongoing development of digital/numeric methods and techniques and to gain in efficiency, a specific study has been initiated. The aim is to analyse what would be the potential benefits of the implementation of numerical techniques in the organization model; would it be better to address the related topics thanks to a dedicated structure (as it is currently performed) or to consider this specific field as a crosscutting topic with its own strategy and associated business plan?

\subsection{Regulatory framework}

Currently, technological developments are due to EDF engineering means and capacities, international cooperation (IAEA, OECD/NEA and World Nuclear Association (WNA)), feedback on various worksites as well as the R\&D research programmes. One of the lessons learned in this particular field is that the efficiency of a technological development depends not only on the techniques used or the operator's ability and mastery but also on regulatory aspects.

Actually, the application of regulations, originally initiated by international directives (IAEA, EC), can vary from one country to another as they are adapted to local considerations and national policies. This point is critical for neighbouring countries as it may result in a risk of confusion in the perception of the public. For instance, some technique performed in one country could be restricted or forbidden in a neighbouring country only due to regulatory constraints, leading to confusion, weakening nuclear operators' credibility as well as lowering public acceptance.

For example, clearance levels, despite a global common European regulation, are not systematically implemented in national policies. This is an illustration of the national policies heterogeneity, preventing from a more efficient circular economy in waste management, at least at the European Union level. 
It would, thus, be advantageous to align and harmonize the application of the internationally agreed regulatory basis. Such a framework shared and applied by all countries with nuclear activities could enable an efficient comparison of the efficiency, the suitability as well as the limits of available techniques being operated in similar situations.

As a consequence, it will become easier to identify the remaining needs of improvements as well as the necessary $\mathrm{R} \& \mathrm{D}$ developments. In addition, a common regulatory basis will help to qualify the decommissioning operators on the common basis of an international shared assessment, which is important as decommissioning is becoming an international business.

\subsection{Financial issues}

Strategic considerations in WM\&D and R\&D have to take into account the cost issues. This includes accurate cost assessment, funding availability, on time, and the associated mechanisms to provide funds.

Since 2006, France, like most other countries, has developed and structured a rigorous and demanding financial system, to guarantee future financing of WM\&D operations. French nuclear operators are on a regular basis audited on their own financial capabilities. The audits include the demonstration of the relevance of the dismantling and waste treatment or conditioning scenarios, the efficiency of the chosen processes as well as the operators' carefulness to maintain them up to date along the years.

\section{Ways and means for R\&D on predisposal activities}

Mainly deduced from EDF observations throughout its decades of experience as worldwide nuclear operator and its strategies developed in WM\&D, 4 ideas for international cooperation are presented here after. They could be the starting point for future R\&D activities at European or international levels in the frame of predisposal activities development.

These preliminary ideas are not exhaustive, as decommissioning and waste management business, being a competitive and emerging market, constitutes a continuous field of innovation and improvements.

\subsection{Alignment of the implementation of the regulatory framework}

Considering the long timeframe and the complexity of the decommissioning activities, the research and innovation effort makes sense if the application of the regulatory framework is similar or at least coherent amongst the countries. For instance, an appropriate definition of criteria and rules for waste management routes may foster the circular economy in material management at European and international level.

Such improvements in regulations should be developed with the support of IAEA and European Commission through their Research and Innovation (R\&I) programmes.
Creating exchange forums will help develop the sharing of experiences and competences. Furthermore, it will enable sensible and relevant comparison of techniques efficiency being performed worldwide.

Alignment of decommissioning objectives, regulatory framework and operating rules will help building confidence among the stakeholders mitigating the risk of confusion in population perception of facility dismantling operations and waste management practices.

\subsection{Improvement of technical and organizational aspects of decommissioning graphite reactors}

In line with its strategy, EDF has already launched the Decommissioning Demonstrator project scheduled to be in operation in 2022. Based on a modular design, the aim of this demonstrator facility is first to check the feasibility of decommissioning scenarios foreseen for graphite reactors. In particular, it will help to improve safety as well as tools and operators' efficiency thanks to an adequate training. This will help to face unexpected situations that will undoubtedly occur during real dismantling as none of the graphite reactors have been designed to be decommissioned. This demonstrator should therefore help to strengthen the technical mastery at appropriate cost. This new flexible facility will enable to test, improve and implement innovative technologies while performing decommissioning on site.

Beyond these objectives, the ambition of the Decommissioning Demonstrator project is to become an international training centre for decommissioning operators. Settled in Chinon (France), this location has the advantage to be at nearly equal distance from most European graphite reactors' locations. This demonstrator will allow operators from foreign countries to share experiences and train themselves on representative mock-ups in safe conditions.

The decommissioning of graphite reactors presents specific characteristics and constraints in terms of scales, dimensions and volumes of waste. Although these specific characteristics are common for all reactors of this type, no real set of decommissioning scenarios and tools are commonly agreed.

Thus, the Decommissioning Demonstrator is a full scale facility with a modular design, ensuring flexibility and adaptability. It will allow the implementation of $R \& D$ projects aimed at testing tools as well as training operators. This facility is composed of two main parts: a 3D simulation platform enabling digitalization and virtual reality and a huge experimental hall with scale one representative mock ups. The hall will serve to improve and qualify innovative techniques, such as remote handling and automated operations.

In addition, the experimental activities aim at improving decommissioning operators' confidence and experience in managing unexpected operational situation. They will also help to determine accurately the types and quantities of secondary induced waste produced in dismantling operation. Moreover, complementing the decommissioning scenarios assessment through this new angle will allow the reduction of waste volume and consequently the limitation of the number 
of waste packages thanks to relevant compromise and choice of tools.

Furthermore, the Decommissioning Demonstrator will allow the development of innovative and applied $\mathrm{R} \& \mathrm{D}$ in an international cooperation framework. The perspective of implementing R\&D based on real cases has already drawn the attention and interests of the United Kingdom and Spain, currently involved in graphite reactors decommissioning.

Supporting countries have been invited by EDF to express their own needs regarding their own decommissioning projects, not limited to graphite reactors, such that they can be taken into account during the design of representative mock-ups, operators training programs, or other topics to be addressed with associated priorities.

The Decommissioning Demonstrator will apply for European support through the 2019-2021 Euratom work programme and in particular through the launch of a specific European Joint Programme (EJP) comprising $\mathrm{R} \& \mathrm{D}$ as well as training activities.

In addition, considering the expression of interest of some members, the OECD/NEA Nuclear Innovative Program (NI2050) has identified this future facility as one of the 12 selected projects of interest in the nuclear field. This OECD/NEA initiative is aiming at accelerating $\mathrm{R} \& \mathrm{D}$ and market deployment of innovative nuclear fission technologies to go with the necessary energy transition towards a very low carbon energy mix.

Finally, the IAEA has recently expressed its willingness to consider such project becoming a formation centre as soon as its industrial objectives will be achieved.

Beyond improving dismantling operation and ongoing waste management (in operation and in decommissioning), the Decommissioning Demonstrator will positively direct the future NPPs design and associated maintenance operations whatever are their nuclear technologies.

\subsection{Development of new techniques to decontaminate/homogenize metallic materials}

Currently, EDF considers the creation, in France, of a new treatment facility to recycle metals. Based on the circular economy principle, this new plant, foreseen to be available by 2030 , will comprise a melting oven and a foundry. The new facility will allow preserving repository capacities thanks to a significant reduction of volumes to be disposed of. It will also preserve source of raw materials such as steel, carbon and nickel.

At the early stage of the project, joint $R \& D$ programmes are designed to establish a sound common technical and regulatory basis to design processes that could accommodate metallic waste coming from various countries. In particular, emphasis will be given to study the use of arc melting furnace technology to decontaminate/ homogenize metallic materials.

When operating, such unique facility could allow centralizing and regulating metallic flows coming from worldwide decommissioning worksites, particularly from Europe and Asia. Metals could therefore be recycled and further reused thanks to this new sustainable industrial process.

\subsection{Increased training of decommissioning operators and implementation of robotics}

A specific emphasis will be put on the need to increase nuclear operators training using automated tools. The training and qualification processes should be established on the common basis of an international shared assessment of current and future practices.

The operators' increased ability and effectiveness will be obtained by strengthening their training on representative configurations and mock-ups specifically designed to take into account the various nuclear technologies constraints and requirements,

For example, the development of remote exoskeleton is foreseen, as human skills and expertise appear irreplaceable in some cases with high dose rate exposure. This R\&D will pave the way for progressively replacing operators by machines without losing human know-how and mastery.

Such improvements and associated R\&D could consequently bring up safety and public acceptance as well as increasing operators' efficiency.

In the future, an operator would be able, safely, from his or her office or a representative mock-up, to operate remotely a humanoid robot present on site. Thanks to 3D and virtual reality, wearing its exoskeleton, the operator will act as if he or she were really operating, adapting his behaviour to the context.

Such evolution in working conditions could lead to several advantages:

- improvements of the labour efficiency (up to $\sim 2$ times), increasing the time of work in conditions of ionized radiation (reduction of the collective dose);

- reductions of personnel's dose exposure;

- dismantlement of larger fragments without necessary heavy handling/lifting means;

- easier worksite preparation with no (or less) need of timeconsuming (and waste-producing) preliminary decontamination;

- reduction of the volumes of secondary induced waste (work clothing, air locks, ...), less washings leading to fewer liquid effluents; and

- a drastic mitigation of the risk of injury, electric shock, falling objects or falling from heights and their consequences.

Last, but not least, it will keep operators' motivation at high level and increase public confidence towards back-end activities by use of cutting-edge technologies. This innovative approach of performing nuclear decommissioning is also foreseen as a means to secure the roadmap by accelerating cutting rates upgrading/modernizing scenarios and technologies. This last characteristic could foster the attractiveness of back-end activities, particularly towards young generations.

\subsection{Intellectual property rights (IPR) issues}

Although IPR is not the prime aim of this paper, it is clear that in a highly technologic area like decommissioning it is important to address IPR since the preliminary phases of discussions, defining $\mathrm{R} \& \mathrm{D}$ programmes. 
Several ways exist to address these topics. They usually depend on the level of involvement of each contributor, their respective internal policies and their will to share their own techniques/know-how aiming to improve them. EDF currently implements various possible IPR schemes. All of them lead to specific agreements, defining respective contributors' commitments. The contractual framework and in particular IPR rules in view of commercial applications should be set specifically for each R\&D project.

\section{Conclusion}

The worldwide experience gained from the last decade at decommissioning worksites lead to a first conclusion: ways and means of R\&D implementation on dismantling activities and waste management are strongly linked to a suitable application of the regulatory framework.

A sound and efficient application will foster a circular economy in materials, will help to establish a common basis of comparison of techniques efficiency and decommissioning operators' ability and will allow waste volume reduction.

This first point is important for identifying the actual remaining lacks and needs of knowledge and consequently the associated $\mathrm{R} \& \mathrm{D}$ developments requirements.
The second point is to put in coherence at the International level operators' training rules (up to their certification). This goal could be achieved on one hand by the sharing of experiences through international cooperation and, on the other hand, by the use of experimental demonstrators equipped with representative mock-ups.

These experimental demonstrators will help to adapt already existing tools or to identify and implement the required applicable pragmatic innovations. It will increase the efficiency of operators and techniques, mitigating risks (dose exposure, human intervention, secondary induced waste (clothing, wash liquid effluents)) and associated costs while securing the global decommissioning roadmap.

The development of sustainable techniques may have a positive impact on public acceptance as well as stakeholders' confidence.

Achieving these objectives will improve future NPPs' design in order to ease future decommissioning operations and thus, strengthening the nuclear industry credibility.

International cooperation, e.g. through the support of the European R\&D work programmes in $W M \& D$ will help to reach a global commitment in a sustainable, internationally shared waste management policy and practices.

Cite this article as: Michel Pieraccini and Sylvain Granger, A nuclear owner/operator perspective on ways and means for joint programming on predisposal activities, EPJ Nuclear Sci. Technol. 6, 20 (2020) 